\title{
Derivation and development of the MacroPics food picture set
}

$3{ }^{+}$Sophie Fromm ${ }^{a, c},{ }^{+}$Emily E. Perszyk ${ }^{a, c}$, Arsene Kanyamibwa ${ }^{\mathrm{a}, \mathrm{c}}$, Kathryn M. Wall ${ }^{\mathrm{a}, \mathrm{c}}$, Zach

4 Hutelin $^{\mathrm{a}, \mathrm{c}}$, Jessica Trinha ${ }^{\mathrm{a}}$, Barry G. Green ${ }^{\mathrm{d}, \mathrm{e}}$, Kyle D. Flack ${ }^{\mathrm{f}}$, Alexandra DiFeliceantonio ${ }^{\mathrm{g}, \mathrm{h}}$, *Dana

$5 \quad$ M. Smalla,b,c

$6+{ }^{+}$Denotes co-first authorship

$8 \quad$ a Modern Diet and Physiology Research Center, New Haven, CT, USA.

$9 \quad$ b Yale University, Department of Psychology, New Haven, CT, USA.

$10{ }^{c}$ Yale University School of Medicine, Department of Psychiatry, New Haven, CT, USA.

$11{ }^{\mathrm{d}}$ Yale University School of Medicine, Department of Surgery, New Haven, CT, USA.

12 e The John B. Pierce Laboratory, New Haven, CT, USA.

$13{ }^{\mathrm{f}}$ University of Kentucky, College of Agriculture, Food, and Environment, Department of

14 Dietetics and Human Nutrition, Lexington, KY, USA.

15 g Virginia Tech, College of Agriculture and Life Sciences, Department of Human Nutrition,

16 Foods and Exercise, VA, USA.

$17{ }^{\mathrm{h}}$ Fralin Biomedical Research Institute, Center for Transformative Research on Health Behaviors, 18 Roanoke, VA, USA.

20 *Correspondence:

21 Dana M Small, PhD

22 dana.small@yale.edu

231 Church Street, Suite 6A

24 New Haven, CT 06510-3330, USA 


\section{Abstract}

26 Recent research suggests that the macronutrient composition of foods can influence

27 reinforcement independent of energy density. To further investigate this phenomenon, we

28 developed "MacroPics," a food picture set depicting processed American snack foods in which

29 items contain: (1) predominantly carbohydrate, (2) predominantly fat, or (3) roughly equal

30 proportions of carbohydrate and fat. We first photographed 60, 120-kcal portions of snack foods

31 falling into one of the three macronutrient categories and asked 61 individuals to rate the images

32 for liking, familiarity, frequency of consumption, healthiness, estimated energy content (in kcal)

33 and expected satiation. A 36-item subset was then selected in an iterative process to minimize

34 differences between the macronutrient categories on these ratings while simultaneously ensuring

35 similar variability within category on a number of food characteristics (e.g., energy density,

36 portion size, retail price) and visual properties (e.g., color and complexity). Item and

37 macronutrient category characteristics of the final MacroPics set are reported.

39 Keywords: Food pictures, food cue reactivity, reinforcement, macronutrient, eating behavior, 40 ingestive behavior 


\section{Introduction}

The food environment has changed markedly over the past 50 years. Not only are there quantitative differences, such as increased availability and energy density, but there are also qualitative differences in the foods we consume, such as combinations and doses of nutrients that have not been encountered previously in hominin evolution (Cordain et al. 2005). Emerging findings suggest that these qualitative changes may affect food choice. For example, DiFeliceantonio et al. (2018) reported that foods containing carbohydrate and fat are more reinforcing than equally liked and isoenergetic foods containing primarily fat or primarily carbohydrate, implying that fat and carbohydrate interact to potentiate reward. Likewise, at the level of physiology, distinct mechanisms exist to relay reinforcing signals derived from fat and carbohydrate from the gut to the brain (Small and DiFeliceantonio 2019; Han et al. 2018; Tellez, Medina, et al. 2013; Tellez, Ren, et al. 2013; Ren et al. 2010). Further understanding the influence of macronutrient content on food decisions and preference is therefore of interest.

In humans, food choice is often studied using food pictures, especially in the neuroimaging environment. Cognitive, behavioral, and neural responses to food images have been shown to mimic those elicited by actual food stimuli (Blechert et al. 2014). As such, results from meta-analyses suggest similar effect sizes when predicting eating- and weight-related outcomes from food-cue reactivity and craving in response to food pictures and real food stimuli (Boswell and Kober 2016). However, although a variety of picture sets are available (Blechert et al. 2019; 2014; King et al. 2018; DiFeliceantonio et al. 2018; Charbonnier et al. 2016), they are not optimized for studying the effects of macronutrients in American samples in several regards. First, picture sets developed in Europe or other countries contain foods that are frequently unfamiliar to Americans (e.g., DiFeliceantonio et al. 2018; Charbonnier et al. 2016). Second, picture sets appropriate for American populations leave many factors uncontrolled. For example, food choice and intake depend on a complex interplay of perceived factors such as palatability, healthiness, estimated energy content (Labbe et al. 2017; Provencher and Jacob 2016), and expected satiation (Guillocheau et al. 2018; Fiszman and Tarrega 2017; Wilkinson et al. 2012; Brogden and Almiron-Roig 2010; Brunstrom and Shakeshaft 2009). Likewise, food characteristics such as total energy content, energy density, or portion size and visual image properties including brightness, color, and contrast are likely to impact responses to food images and are not equated across different macronutrient types (Blechert et al. 2014). Therefore, the 
72 aim of the current study was to develop a picture set of processed American snack foods in

73 which items contain: (1) predominantly carbohydrate, (2) predominantly fat, or (3) roughly equal

74 proportions of carbohydrate and fat, while also minimizing differences between these

75 macronutrient categories on food characteristics (e.g., energy density, portion size, retail price),

76 visual properties (e.g., color and visual space taken up by the food on the plate), and perceived

77 ratings of liking, familiarity, frequency, healthiness, estimated energy content and expected

78 satiation.

\section{2. Materials and Methods}

2.1 Participants

Sixty-one healthy, English-speaking participants aged 18-45 years took part in the study.

82 Sample size was oriented towards a prior study aimed to establish a similar picture set for the

83 German population (DiFeliceantonio et al. 2018). To qualify for the study, participants were

84 required to have lived in the United States for the past five years without any interruption of

85 more than nine months to ensure prior exposure to American snack foods. Other exclusion

86 criteria included: a) serious or unstable medical illness; b) current or history of major psychiatric

87 illness as defined by the DSM-IV criteria; c) medications that affect alertness, any psychoactive

88 drugs or anti-obesity agents; d) known taste or smell dysfunction; e) a diagnosis of diabetes; f)

89 food allergies, food sensitivity or dietary restriction for other reasons (religious, choice, etc.); g)

90 current pregnancy/nursing. Eligibility was determined via online questionnaires and follow-up

91 email conversation. On the study day, the severity of depression and anxiety symptoms was

92 assessed by means of the Beck Depression Inventory II (BDI-II; A. Beck et al. 1996) and the

93 Beck Anxiety Inventory (BAI; A. T. Beck et al. 1988), respectively. Altered eating behavior can

94 be one symptom of depression or anxiety (Rosenbaum and White 2015; Mooreville et al. 2014;

95 Frost et al. 1982; Herman and Polivy 1975), so we had planned to exclude participants with

96 scores indicative of severe depression or anxiety post-hoc from the analysis. No participants

97 were excluded on this basis. The experimental protocol was approved by the Yale Human

98 Investigation Committee and all participants provided signed, informed consent.

100 Initial pictures were obtained for 60 processed snack foods selected by a multi-

101 disciplinary team of psychologists, neuroscientists, and nutritionists familiar with typical 
102 American eating habits. All images were taken in-house with a professional camera and photo

103 light box. Each picture contained a 120-kcal food portion arranged in the middle of a white plate

104 as shown in Figure 1A. To provide visual cues for portion size, a paper cup and napkin were

105 placed next to the plate in each photo. All images were color photographs with dimensions of

$1065760 \times 3840$ pixels and a resolution of 300 pixels/inch.

$107 \quad 2.2 .1$ Food characteristics

108 Data on food composition (energy density, total portion size, and gram $\{\mathrm{g}\}$ or milligram

$109\{\mathrm{mg}\}$ per serving of fat, carb, protein, sodium, or water) were collected using the Nutrition Data

110 System for Research (NDSR) software version 2018, developed by the Nutrition Coordinating

111 Center (NCC), University of Minnesota, Minneapolis, MN. The NDSR provides a complete

112 nutrient profile for all foods in the database. Data on foods not included in the database were

113 calculated using the nutrient content of other nutrients in the same food, a product ingredient list,

114 or estimates from similar foods. All food composition metrics from the NDSR in g or mg per

115 serving were converted to $\mathrm{g}$ or $\mathrm{mg}$ per $120 \mathrm{kcal}$ (equivalent to the amount shown in each image).

116 Food volume in $\mathrm{cm}^{3}$ was estimated by using displacement of poppy seeds similar to the method

117 from Segnini et al. (2004). Poppy seeds were first packed into a $250 \mathrm{~mL}$ glass beaker. Another

118 glass beaker of the same size was then filled with $40 \mathrm{kcal}$ of a given food in addition to the

119 poppy seeds from the first beaker. Any seeds that overflowed (i.e., no longer fit in the allotted

120 volume) were collected and measured in $\mathrm{mL}$, which was converted to $\mathrm{cm}^{3}$ and multiplied by

121 three for the volume of $120 \mathrm{kcal}$ of each food. Price in dollars per $120 \mathrm{kcal}$ was determined from

122 unit prices $(\$ / \mathrm{g})$ at Walmart.com using the IP address at the lab where all research was

123 conducted.

$124 \quad 2.2 .2$ Visual properties

125 Visual area, or space taken up by food in the 2-dimensional image (not accounting for 126 food overlap), was calculated using Image J 1.x (Schneider, Rasband, and Eliceiri 2012) first in

127 pixels and converted to $\mathrm{cm}^{2}$ based on the measured dimensions of the plate. To prevent

128 confounding by low-level visual input, image characteristics were also taken into consideration

129 in the selection of the final picture set. These image characteristics included red, green, and blue

130 color, brightness, within-object contrast, spatial frequency, and complexity, and were computed

131 and normalized using available Matlab R2018b (The Mathworks Inc., Sherborn, MA, USA) 
132 scripts from Blechert et al. (2014). Details on quantifying these image characteristics can also be

133 found in Blechert et al. (2014).

$134 \quad 2.2 .3$ Perceptual ratings

135 All rating questions and anchors are described in Table 1. We assessed liking, familiarity, 136 estimated energy content, and estimated energy density following a prior study that evaluated a

137 similar food picture set for the German population (DiFeliceantonio et al. 2018). Additionally, 138 we asked participants to rate frequency of consumption, perceived healthiness, and expected 139 satiation. Frequency of consumption was added as another measure of familiarity, framed to 140 inquire explicitly the ingested exposure of the presented food items. Perceived healthiness

141 (Provencher and Jacob 2016) and expected satiation (Brunstrom et al. 2018; Forde, Almiron-

142 Roig, and Brunstrom 2015; Brunstrom and Rogers 2009) are foci of recent research and have

143 been shown to impact food choice and meal size selection. Therefore, we included both variables

144 to satisfy any interest of potential researchers that may use this food picture set or a subset of its

145 images in the future.

$146 \quad 2.3$ Additional Measures

$147 \quad$ 2.3.1 Eating behavior

148 The Dutch Eating Behavior Questionnaire (DEBQ; van Strien et al. 1986) was used to 149 assess eating behavior. The DEBQ comprises 33 items to be answered on a five-point scale, and 150 these items are summed to obtain factor scores of restrained, emotional, and external eating. This 151 three-factor structure of the DEBQ has been confirmed among multiple samples and languages, 152 including English (Wardle 1987) with internal consistency for all subscales estimated at an inter 153 class coefficient (ICC) $\geq .79$ (van Strien et al. 1986).

$154 \quad$ 2.3.2 Dietary intake

155 The Dietary Fat and Free Sugar - Short Questionnaire (DFS; Francis and Stevenson 1562013 ) is a 26-item checklist that we used to assess the frequency (monthly intake) of fatty and 157 sugary foods across our participants. Items are rated on a five-point scale and summed to a 158 composite score. Test-retest reliability has been estimated at ICC $=.83$. Correlations with free 159 sugar and fat intake evaluated via a large established food frequency questionnaire and four-day 160 dietary record were estimated at $r=.35-.71$ (Francis and Stevenson 2013). Our picture set 
161 contains fat, carbohydrate (carb), and combo foods, and so we selected the DFS for a measure of

162 frequency of consumption specific to these macronutrient types.

1632.4 Procedure

164 Participants were instructed to arrive at the laboratory neither hungry nor full, but at least

165 1-hr fasted. After obtaining signed informed consent, we asked participants about compliance

166 with fasting; all participants reported compliance. Participants were trained on using our rating

167 scales (Table 1) and received verbal explanations of the terms in the rating scales, specifically

168 "calorie content" (i.e., energy content) and "energy density" to ensure that they understood the

169 difference. Calorie content was described as "a unit of energy that refers to the amount of energy

170 people get from the foods or drinks they consume" and energy density as "the number

171 of calories stored in the food per unit volume." We gave examples for both constructs and

172 participants had the opportunity to ask further questions. Participants then rated their 'internal

173 state' on five items (Table 1). These ratings were used to verify that participants arrived at the

174 laboratory neither hungry nor full (on average). Next, we presented participants with all 60 food

175 image stimuli in a digital task designed in PsychoPy version 3.0 (Peirce 2007) for Python.

176 Participants completed the seven perceptual ratings for each picture on continuous visual analog 177 scales (VAS), with the exception of liking on the Labeled Hedonic Scale (Lim et al. 2009). Food 178 images were presented in a randomized order for each participant. Subsequently, participants

179 provided demographic information (e.g., age, gender, race, education level) and completed

180 digital versions of the DFS, DEBQ, BDI and BAI. Lastly, we collected anthropometric data of 181 height, weight, waist circumference, hip circumference, body fat percentage, and BMI. Height

182 was obtained to the nearest centimeter and weight to the nearest hundredth of a kilogram by 183 means of digital scales. Waist and hip circumference were measured to the nearest centimeter

184 using a tape measure. Body fat percentage was computed via bio-electrical impedance analysis

185 (Seca Medical Body Composition Analyzer mBCA 515, Hamburg, Germany) to the nearest

186 tenth of a percent. BMI was calculated as weight divided by the square of height (in $\left.\mathrm{kg} / \mathrm{m}^{2}\right)$ to

187 the nearest hundredth. Each session lasted 1 to 1.5 hours and participants were compensated 188 upon completion.

\subsection{Picture selection and data analysis}


All perceptual ratings (aside from frequency in days/week, liking on the LHS, and estimated energy content in kcal) for individual food items were scored as a decimal from 0-1

192 based on position along the 10-cm VAS. Data processing was carried out with Matlab R2018b

193 (The MathWorks Inc., Sherborn, MA). After study completion, we selected 36 pictures of fat, 194 carb, and combo foods (12 food items per category). Items were selected into the final picture set 195 such that macronutrient categories would minimally differ on the food characteristics, visual 196 properties, and perceptual ratings described above. In a first step, we selected items in a way that 197 food categories did not differ on food characteristics, with the exception that differences in fat 198 and carbohydrate content were present according to the fat, carb, and combo category

199 classifications. In a second step, we checked that these categories did not differ on visual 200 characteristics. Using MATLAB's built-in outlier function, we identified items that drove the 201 differences between the categories and iteratively exchanged them, simultaneously checking that 202 categories differed minimally on the previously matched food characteristics. Lastly, we

203 followed the same steps to ensure that as many of the perceptual ratings as possible would be 204 similar across the three macronutrient categories. All category differences were computed with 205 one-way ANOVAs on average values per food item. Post-hoc t-tests were conducted for carb, 206 fat, and protein content as well as for within-object contrast and estimated energy density using 207 alpha levels of .0167 that were Bonferroni-adjusted for the number of comparisons (.05/3). All 208 data were plotted in GraphPad Prism version 8.2.0 (GraphPad Software, La Jolla, California, 209 USA). In the reported plots, each data point represents the average for a food item (12 items per 210 macronutrient category, or 36 items in total). Lastly, as secondary analyses, we examined

211 correlations between mean subjective ratings of each participant with individual differences in

212 terms of age, BMI, body fat percentage, hunger ratings as well as with scores on our measures of 213 eating behavior and fat and sugar consumption. There were no missing data points and no

214 excluded participants. The full analytic plan was developed prior to data collection.

\section{3. Results}

\subsection{Participants}

217 Participant characteristics of the final sample $(\mathrm{N}=61)$ are summarized in Table S1. A 218 summary of participant internal state ratings is shown in Table S2. Although there was 219 participant-to-participant variation, it is important to note that the mean internal state rating of 220 hunger was $0.52 \pm 0.25)$ and of fullness was $0.38 \pm 0.24(M \pm S D)$, suggesting that participants 
221 arrived neither hungry nor full, as instructed.

222 3.2 Final 36-item picture set "MacroPics"

Food items in the full 60-item picture set and in the final 36-item picture set for macronutrient-based comparisons are reported in Table 2 with example food image stimuli

225 depicted in Figure 1. All images portrayed 120-kcal portions in order to keep actual energy content constant. A comparison of food characteristics across macronutrient categories of the selected picture set are presented in Table 3 and Figure 2A. Macronutrient categories did not significantly differ on food characteristics other than fat, carb, and protein content. We were unable to keep protein content similar across the categories and post-hoc tests revealed that protein content in the fat category was greater compared to the carb $(F(1,22)=39.67, p \leq .001)$ and the combo category $(F(1,22)=55.307, p \leq .001)$, but no difference existed between the carb and combo categories $(F(1,22)=0.88, p=.77)$. Intentionally, the carb category contained significantly less fat than the fat category $(F(1,22)=819.252, p \leq .001)$. The combo category $(F$ $(1,22)=149.87, p \leq .001)$ and fat category $(F(1,22)=52.39, p \leq .001)$ contained significantly more fat than the carbohydrate category and less carbohydrate than the carb $(F(1,22)=818.286$, $\mathrm{p} \leq .001)$ and the combo category $(F(1,22)=282.559, p \leq .001)$. The combo category contained more carbohydrate than the fat category $(F(1,22)=107.296, p \leq .001)$. for within-object contrast (Table 4, Figure 2B). However, post-hoc tests revealed no significant differences in within-object contrast among pairwise comparisons of the carb versus fat ( $F(1$, $22)=5.54, \mathrm{p}=.084)$, carb versus combo $(F(1,22)=0.02, \mathrm{p}=1.00)$, or fat versus combo $(F(1$, $22)=6.63, p=.052$ ) categories. Finally, the only significant difference observed across

243 macronutrient categories in perceptual ratings was for estimated energy density (Table 5, Figure

244 2C). Participants rated the fat category to have significantly greater estimated energy density 245 than the carb category $(F(1,22)=16.59, \mathrm{p}<.01)$, but not the combo category $(F(1,22)=6.52$, $246 \mathrm{p}=.068)$. There was also no significant difference in estimated energy density between the carb 247 and combo categories $(F(1,22)=1.72, \mathrm{p}=.466)$. Food characteristics, visual properties, and 248 perceptual ratings of all individual food stimuli (including those in the initial 60-item set but not 249 the final 36-item 'controlled' set) can be found in Tables S3-S5, respectively. 
As presented in Table 6, we found no associations of BMI or of hunger score with any of

252 the rating variables. Body fat percentage was lower in participants that indicated they ate the

253 presented snacks more often. Older participants indicated greater food familiarity. Participants

254 with greater fat and sugar consumption measured with the DFS reported greater liking and

255 frequency of consumption of our items. Participants with high restraint and external eating in the

256 DEBQ reported greater liking of our food items. Emotional eating was not related to any of the

257 subjective ratings.

\section{4. Discussion}

259 Here we report the development of "MacroPics," a 36-item picture set depicting

260 American snack foods that are made primarily from: (1) carbohydrate, (2) fat, or (3) a

261 combination of carbohydrate and fat. By design, the food images all show 120-kcal portions and

262 were selected to create three macronutrient categories differing minimally on a number of food

263 characteristics (e.g., nutrient composition, volume, and price), visual properties (e.g., visual area,

264 colors, and intensity), and subjective ratings (e.g., liking, familiarity, and estimated energy

265 content). This strategy optimizes the picture set for testing hypotheses in U.S.-based samples,

266 where the choice, perception, and/or value of the food item may depend upon macronutrient

267 content. Given the accumulating evidence that distinct physiological mechanisms support the

268 reinforcement value of fat compared with carbohydrates in both humans (Small and

269 DiFeliceantonio 2019) and rodents (Han et al. 2018; Zhang et al. 2018; Tellez, Medina, et al.

270 2013), we anticipate that this picture set will be useful for addressing emerging questions.

271 MacroPics was developed to parallel a food picture set used in a German sample to show

272 that people bid more for isoenergetic and similarly liked foods that contain both fat and

273 carbohydrate compared to fat or carbohydrate alone (DiFeliceantonio et al. 2018). However,

274 MacroPics presents several strengths over the earlier version. First, it includes measures of food

275 volume, visual area of the food, and actual price based on a national grocery provider. In

276 addition, we obtained perceptual ratings for frequency of food consumption and expected

277 satiation. The former is similar to familiarity but more directly assesses intake rather than visual

278 exposure. The latter serves as a read-out of the feeling of fullness that is learned or remembered

279 from prior experience eating a given food. Second, our food images all contain identical portion

280 calories $(120 \mathrm{kcal})$, rather than approximate amounts that vary from stimulus-to-stimulus. Third, 
281 in contrast to large online databases of food image stimuli, our food items are each portrayed on

282 an identical 'standardized' plate, alongside a paper cup and napkin. Our stimuli thus contain

283 actual food in a realistic presentation style, as opposed to the plain white background or

284 photoshopped colors often employed in images that are gathered from varying internet sources

285 (DiFeliceantonio et al. 2018; Blechert et al. 2019; 2014; King et al. 2018). Fourth, perceptual

286 ratings for all images were made by participants in the same laboratory setting, rather than

287 through online surveys (e.g., Blechert et al. 2019; 2014; Prada et al. 2017 for a Portuguese

288 validation). While this means our overall sample size may be smaller and limited to Americans,

289 we obtained consistent ratings from all participants (rather than ratings for each food from a

290 subset of the sample). Along the same lines, we were able to instruct participants to arrive neither

291 hungry nor full (but at least 1-hr fasted) and could confirm through self-reported ratings during

292 our in-person session that fasting was roughly consistent across our sample. Lastly, in addition to

293 the ANOVA statistics for our final 36-item picture set (of carb, fat, and combo categories), we

294 report here the food characteristics, visual properties, and perceptual ratings for all 60 food

295 images used in our study. We are working further to develop two additional versions of

296 MacroPics, one with multiple portion sizes for each food item, and another that presents the

297 items without the plate, napkin, and cup (with only a gray background). Early examples of these

298 future stimulus types are displayed in Figure S1.

Although we were able to minimize differences between the macronutrient categories, there were several variables that could not be fully equated. These variables are summarized in

301 Table 7. Most notably, the average protein content of the fat items was significantly greater than

302 that of the carb and combo items and sodium followed the same trend. Here the challenge

303 resulted from a limited selection of fat-containing snack foods that do not also contain great

304 amounts of protein or sodium. Current evidence that the valuation of protein is distinct from

305 carbohydrate and fat is limited to binary-choice trials of pairs of relatively healthy and

306 unprocessed foods (e.g., fruits, vegetables, meats, and grains) differing in nutrient composition

307 (Buckley et al. 2019). Similar explorations are warranted for the role of protein in the reward 308 value of highly processed snack foods.

309 With respect to visual properties, within-object contrast differed across the categories, 310 though pairwise tests corrected for multiple comparisons were not significant. We also found 311 trend-level category differences in brightness, the color blue, visual area covered by the food, 
312 and volume (listed as a food characteristic in Table 7). Since foods with larger visual area or

313 volume are at particular risk of eliciting greater desire to eat (Burger et al. 2011), visual area and

314 volume should be accounted for in future studies using MacroPics. Among our perceptual

315 ratings, participants estimated significantly greater energy density of fat compared with carb

316 items. A trend in liking was also observed. Greater liking of combo foods as our trend shows is

317 consistent with prior work (DiFeliceantonio et al. 2018) and made it particularly challenging to

318 minimize the difference in liking across our categories. Considering the number of variables that

319 we aimed to keep similar, we had to accept these differences in favor of other variables.

320 Researchers are encouraged to consider these factors when using the MacroPics picture set and

321 may elect to use those variables which are trending or different among the macronutrient

322 categories (Table 7) as covariates in their analyses. We have highlighted those which we believe

323 are most important to include as covariates per the reasons above (Table 7).

324 There are also other caveats to keep in mind with MacroPics. First, it includes only

325 processed snack foods. These foods are more likely to contain added products (especially sugars)

326 and are ubiquitous in US grocery stores. A recent survey of a representative sample of over 9000

327 individuals concluded that, on average, over half of American daily energy intake comes from

328 ultra-processed foods (Steele et al. 2016). Moreover, while there appears to be a link between

329 obesity and consumption of processed foods (Louzada et al. 2015; Canella et al. 2014; Asfaw

330 2011), it remains unclear whether this may be a consequence of 'processing' itself, or of the

331 modified macronutrient content in these foods (Poti et al. 2017). MacroPics may help to

332 disentangle these possibilities. Future work may further benefit from the creation of picture sets

333 using full meals or healthy snacks that could be better matched on protein. Second, perceptual

334 ratings were obtained from participants who were at least 1-hr fasted. Varying the length of food

335 restriction prior to testing may alter subjective ratings of the stimuli used here. For example,

336 longer food deprivation renders energy dense foods more attractive than their less energy dense

337 counterparts (Goldstone et al. 2009). Although our images each show 120-kcal portions, it is

338 possible that fasting may modulate food reward according to participant ratings of estimated 339 energy content or density.

340 Our picture set provides researchers with a tool to study human food perception and 341 macronutrient-based responses to food stimuli, including, but not limited to food liking, expected 342 satiation, understanding of portion, reward value, craving, cue reactivity, and emotional valence. 
343 The food picture approach is of particular interest for neuroimaging studies in which exposure to

344 real food is not feasible in the fMRI scanner and/or a highly controlled set of stimuli is

345 necessary. MacroPics images and materials are fully and freely available online at the Modern

346 Diet and Physiology Research Center website (mdprc.org/resources).

348 Conflict of Interest Statement: The authors declare no competing commercial or financial 349 interests.

351 Statement of Ethics: The study was conducted according to the standards laid down in the

352 Declaration of Helsinki. All procedures were approved by the Yale Human Investigation

353 Committee. All participants provided signed consent to participate in the study.

Data Availability: The following are available at mdprc.org/resources or from the corresponding author upon request: (1) all 60 food images at $120 \mathrm{kcal}$, (2) all data used to support the findings of the study, and (3) Matlab scripts for analyses.

Acknowledgements: This work was supported by the Modern Diet and Physiology Research

360 Center.

362 Authors' contributions: SF, EEP, KMW, BGG, KDF, AD, and DMS contributed to conception 363 and design of the study; KMW, SF, JT, and BGG photographed food pictures; ZH, JT, and KDF 364 collected food composition data; SF and AK collected participant data; SF, EEP, ZH, and AD 365 performed the statistical analysis; SF and EEP generated tables and figures; SF, EEP, and DMS 366 wrote the original manuscript draft; DMS acquired the funding to support the study. All authors 367 contributed to manuscript revision and read and approved the submitted version. 


\section{$368 \quad$ Figures}

Carb

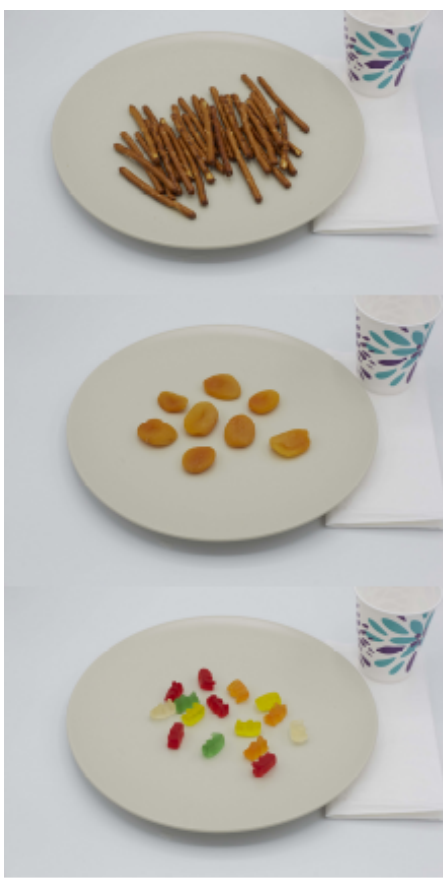

Fat

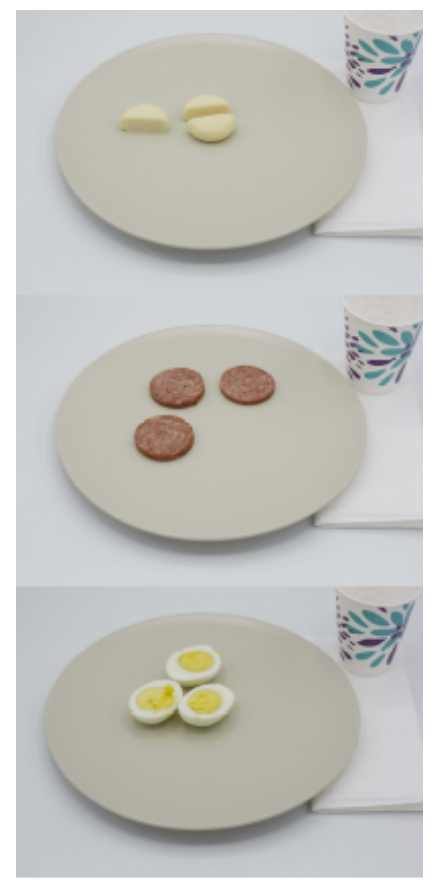

Combo

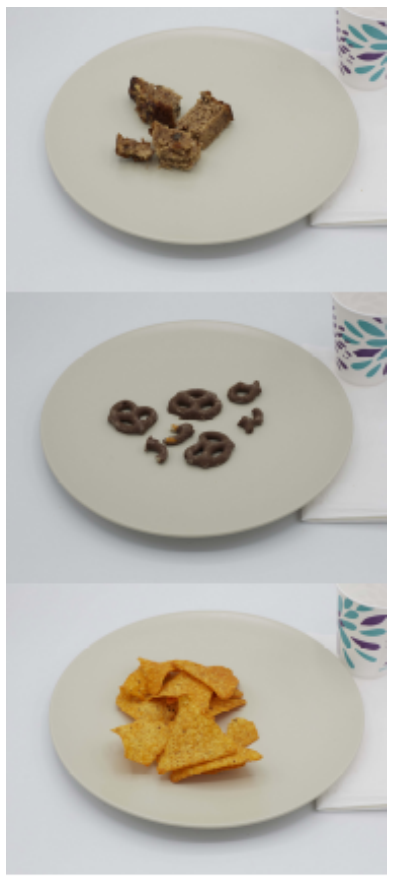

370 Figure 1. Example MacroPics images selected into the final 36-item picture set. Items were

371 balanced into three macronutrient-based categories: carbohydrate ("carb"), fat, and the

372 combination of carbohydrate and fat ("combo"). 

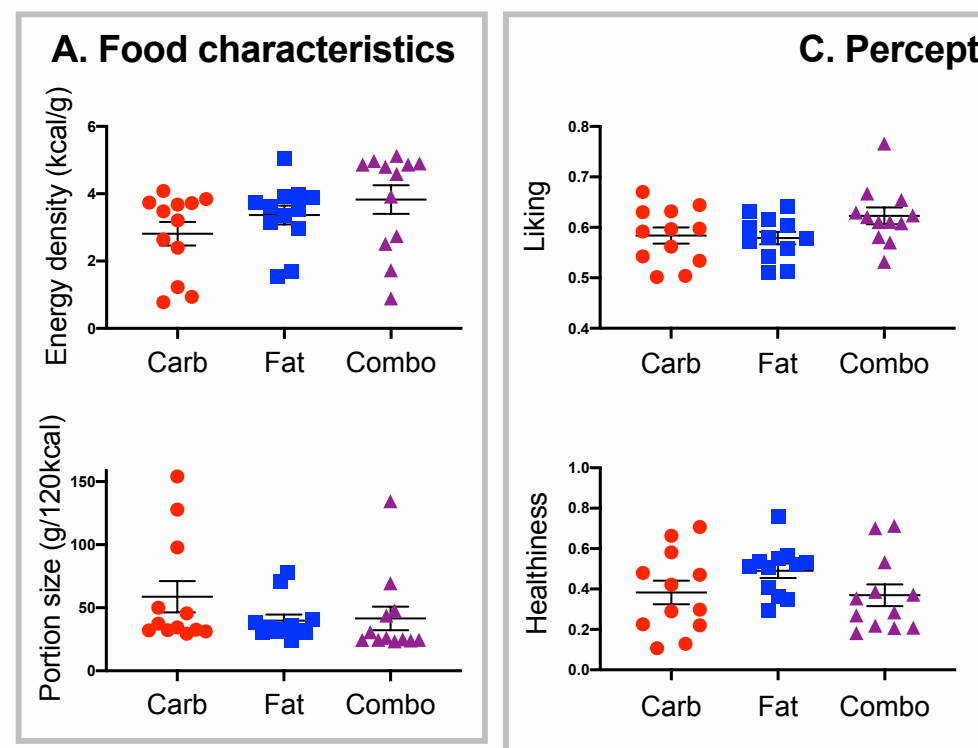

\section{ual ratings}
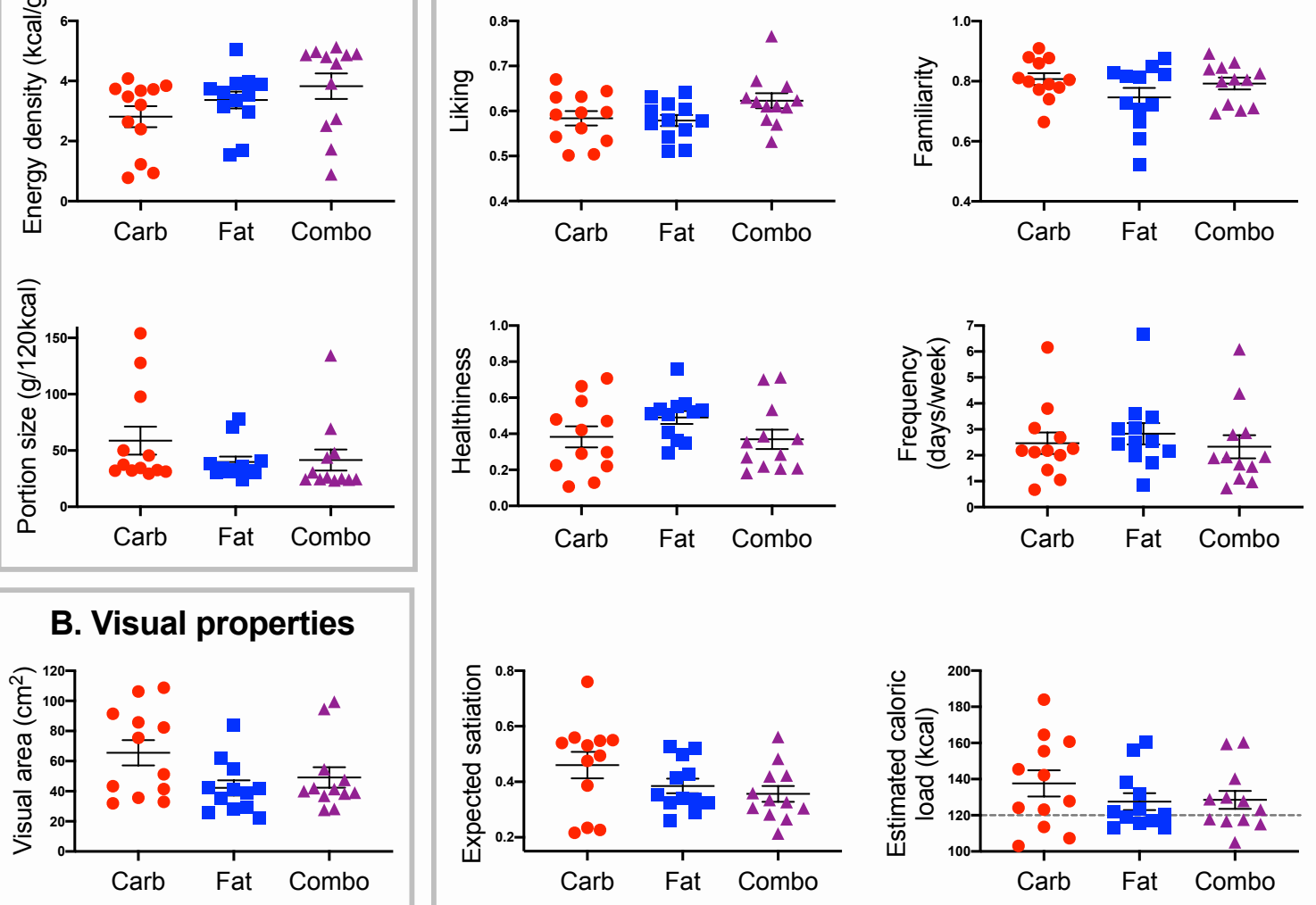

374 Figure 2. Mean \pm SEM of selected (A) food characteristics, (B) visual properties, and (C)

375 perceptual ratings across macronutrient categories of the final MacroPics 36-item picture set.

376 The gray dotted horizontal line in the plot for estimated energy content depicts the actual energy

377 content of all items shown $(120 \mathrm{kcal})$. Each point represents the average of a food item in that 378 category. 
381 Table 1. Anchors and descriptions of internal state ratings and food perceptual ratings. Note that

382 all scales were continuous with ratings possible anywhere along a horizontal or vertical VAS.

\begin{tabular}{|c|c|c|}
\hline \multicolumn{3}{|c|}{ Internal state ratings } \\
\hline Label & Question & Anchors \\
\hline Hunger & How hungry do you feel? & not at all hungry, extremely hungry \\
\hline Thirst & How thirsty do you feel? & not at all thirsty, extremely thirsty \\
\hline Fullness & How full do you feel? & not full at all, extremely full \\
\hline Potential to eat & $\begin{array}{l}\text { How much do you think you could eat } \\
\text { right now? }\end{array}$ & extremely large amount, not another bite \\
\hline Desire to eat & How much do you want to eat right now? & not at all, extremely \\
\hline \multicolumn{3}{|c|}{ Food perceptual ratings } \\
\hline Label & Question & Anchors \\
\hline Liking & Labeled Hedonic Scale (Lim et al. 2009) & see reference \\
\hline Familiarity & How familiar is this food? & extremely unfamiliar, extremely familiar \\
\hline Expected satiation & $\begin{array}{l}\text { How filling do you expect this food } \\
\text { portion to be? }\end{array}$ & not filling at all, extremely filling \\
\hline $\begin{array}{l}\text { Frequency of } \\
\text { consumption }\end{array}$ & How often do you eat this food? & $\begin{array}{l}<1 \text { per month, } 2-3 \text { per month, } 1-2 \text { per } \\
\text { week, } 3-4 \text { per week, } 5+\text { per week }\end{array}$ \\
\hline Healthiness & How healthy is this food? & extremely unhealthy, extremely healthy \\
\hline $\begin{array}{l}\text { Estimated energy } \\
\text { content }\end{array}$ & How many calories are in this portion? & $0,60,120,180,240$ \\
\hline Energy density & How energy-dense is this food? & extremely low, extremely high \\
\hline
\end{tabular}


385 Table 2. Food item list for each macronutrient category, with items of the selected picture set 386 presented in the upper section and unselected items in the lower section.

\begin{tabular}{|c|c|c|}
\hline Carb & Fat & Combo \\
\hline Bagel & American cheese & Banana nut bread \\
\hline Baked beans & Babybel cheese wheels & Cheese and crackers \\
\hline Dried apricots & Blue cheese & Chocolate covered pretzels \\
\hline Froot loops & Breakfast sausage & Chocolate raisins \\
\hline Frosted flakes & Brie cheese & Doritos \\
\hline Fruit snacks & Colby jack cheese & Guacamole \\
\hline Gummy bears & Deviled eggs & Mini nutter butters \\
\hline Jelly beans & Hardboiled eggs & Mini chocolate chip cookies \\
\hline Lucky charms & Pepperoni & Pizza rolls \\
\hline Pineapple rings & String cheese & Peanut butter crackers \\
\hline Pretzels & Summer sausage & Pringles \\
\hline Sorbet & Swiss cheese & Roasted red pepper hummus \\
\hline Life savers & Castelvetrano olives & Apple pie \\
\hline Lollipops & Kalamata olives & Blueberry muffin \\
\hline Marshmallows & Smoked salmon (lox) & Croissant \\
\hline Raisins & Stuffed pimiento olives & Donuts \\
\hline Rice cakes & Vienna sausage & Donut holes \\
\hline Sour gummy worms & & French fries \\
\hline \multirow[t]{6}{*}{ Strawberry licorice } & & Ice cream \\
\hline & & Mini oreos \\
\hline & & Pizza \\
\hline & & Popcorn chicken \\
\hline & & Ramen noodles \\
\hline & & Snickers \\
\hline
\end{tabular}


389 Table 3. Descriptive statistics and one-way ANOVAs on food characteristics of images across

390 macronutrient categories

391

\begin{tabular}{|c|c|c|c|c|}
\hline \multirow{2}{*}{$\begin{array}{l}\text { Food } \\
\text { characteristics }\end{array}$} & Carb & Fat & Combo & \multirow{2}{*}{$\begin{array}{l}\text { Category } \\
\text { difference }\end{array}$} \\
\hline & Mean \pm SEM, Range & Mean \pm SEM, Range & Mean \pm SEM, Range & \\
\hline $\begin{array}{l}\text { Carb } \\
(\mathrm{g} / 120 \mathrm{kcal})\end{array}$ & $28.33 \pm 0.94,23.83-31.81$ & $0.98 \pm 0.20,0.16-2.26$ & $15.36 \pm 0.83,10.46-20.96$ & $\begin{array}{l}\mathrm{F}(2,33)=349.41, \\
p \leq .001 *\end{array}$ \\
\hline $\begin{array}{l}\text { Fat } \\
(\mathrm{g} / 120 \mathrm{kcal})\end{array}$ & $0.47 \pm 0.13,0.02-1.19$ & $9.72 \pm 0.29,8.01-11.01$ & $5.96 \pm 0.43,4.54-9.55$ & $\begin{array}{l}\mathrm{F}(2,33)=225.72 \\
p \leq .001^{*}\end{array}$ \\
\hline $\begin{array}{l}\text { Sodium } \\
(\mathrm{mg} / 120 \mathrm{kcal})\end{array}$ & $142.69 \pm 50.46,1.36-558.44$ & $291.33 \pm 34.46,97.06-523.94$ & $188.82 \pm 39.65,10.81-535.38$ & $\begin{array}{l}\mathrm{F}(2,33)=3.27 \\
\mathrm{p}=.051\end{array}$ \\
\hline $\begin{array}{l}\text { Water } \\
(\mathrm{g} / 120 \mathrm{kcal})\end{array}$ & $27.21 \pm 12.18,0.81-121.72$ & $20.98 \pm 4.66,6.79-57.94$ & $17.87 \pm 9.13,0.57-108.38$ & $\begin{array}{l}\mathrm{F}(2,33)=0.27 \\
\mathrm{p}=.770\end{array}$ \\
\hline $\begin{array}{l}\text { Portion size } \\
(\mathrm{g} / 120 \mathrm{kcal})\end{array}$ & $58.72 \pm 12.43,29.39-154.09$ & $39.77 \pm 4.80,23.79-77.65$ & $41.54 \pm 9.36,23.41-134.43$ & $\begin{array}{l}F(2,33)=1.24 \\
p=.303\end{array}$ \\
\hline $\begin{array}{l}\text { Volume } \\
\left(\mathrm{cm}^{3}\right)\end{array}$ & $88.38 \pm 16.64,25.50-187.50$ & $53.77 \pm 7.57,30.06-120.90$ & $57.20 \pm 8.83,30.60-137.40$ & $\begin{array}{l}F(2,33)=2.65 \\
p=.086\end{array}$ \\
\hline $\begin{array}{l}\text { Price } \\
(\$ / 120 \mathrm{kcal})\end{array}$ & $0.55 \pm 0.03,0.22-1.23$ & $0.65 \pm 0.02,0.25-1.03$ & $0.59 \pm 0.05,0.18-2.13$ & $\begin{array}{l}F(2,33)=0.190 \\
p=.827\end{array}$ \\
\hline
\end{tabular}


393 Table 4. Descriptive statistics and one-way ANOVAs on visual properties of images across 394 macronutrient categories

395

\begin{tabular}{|c|c|c|c|c|}
\hline \multirow{2}{*}{$\begin{array}{l}\text { Visual } \\
\text { properties }\end{array}$} & Carb & Fat & Combo & \multirow{2}{*}{$\begin{array}{l}\text { Category } \\
\text { difference }\end{array}$} \\
\hline & Mean \pm SEM, Range & Mean \pm SEM, Range & Mean \pm SEM, Range & \\
\hline $\begin{array}{l}\text { Visual area of } \\
\text { food }\left(\mathrm{cm}^{2}\right)\end{array}$ & $65.54 \pm 8.41,31.98-108.72$ & $42.21 \pm 5.06,22.4-84.05$ & $49.18 \pm 6.79,27.66-99.49$ & $\begin{array}{l}F(2,33)=3.021, \\
p=.062\end{array}$ \\
\hline Red & $0.34 \pm 0.00,0.34-0.35$ & $0.34 \pm 0.00,0.34-0.35$ & $0.34 \pm 0.00,0.34-0.36$ & $\begin{array}{l}F(2,33)=1.97 \\
p=.156\end{array}$ \\
\hline Green & $0.34 \pm 0.00,0.33-0.34$ & $0.34 \pm 0.00,0.33-0.34$ & $0.34 \pm 0.00,0.33-0.34$ & $\begin{array}{l}F(2,33)=0.36 \\
p=.698\end{array}$ \\
\hline Blue & $0.32 \pm 0.00,0.31-0.33$ & $0.33 \pm 0.00,0.32-0.33$ & $0.32 \pm 0.00,0.31-0.33$ & $\begin{array}{l}F(2,33)=2.57 \\
p=.092\end{array}$ \\
\hline Brightness & $65.25 \pm 1.61,55.67-76.20$ & $63.43 \pm 1.38,58.32-71.02$ & $69.94 \pm 2.59,61.12-87.04$ & $\begin{array}{l}F(2,33)=3.02 \\
p=.063\end{array}$ \\
\hline $\begin{array}{l}\text { Within-object } \\
\text { contrast }\end{array}$ & $27.96 \pm 1.57,19.74-40.60$ & $23.04 \pm 1.38,18.93-35.59$ & $27.69 \pm 1.17,20.76-34.30$ & $\begin{array}{l}\mathrm{F}(2,33)=4.00 \\
p=.028^{*}\end{array}$ \\
\hline $\begin{array}{l}\text { Spatial } \\
\text { frequency }\end{array}$ & $14.64 \pm 0.20,13.41-15.55$ & $14.66 \pm 0.18,15.48-17.19$ & $14.98 \pm 0.10,14.53-15.53$ & $\begin{array}{l}F(2,33)=1.35 \\
p=.274\end{array}$ \\
\hline Complexity & $0.02 \pm 0.00,0.01-0.04$ & $0.02 \pm 0.00,0.01-0.03$ & $0.02 \pm 0.00,0.01-0.04$ & $\begin{array}{l}F(2,33)=2.43 \\
p=.104\end{array}$ \\
\hline
\end{tabular}


397 Table 5. Descriptive statistics and one-way ANOVAs on perceptual ratings of images across

398 macronutrient categories

399

\begin{tabular}{|c|c|c|c|c|}
\hline \multirow{2}{*}{$\begin{array}{l}\text { Perceptual } \\
\text { ratings }\end{array}$} & Carb & Fat & Combo & \multirow{2}{*}{$\begin{array}{l}\text { Category } \\
\text { difference }\end{array}$} \\
\hline & Mean \pm SEM, Range & Mean \pm SEM, Range & Mean \pm SEM, Range & \\
\hline Liking & $0.58 \pm 0.02,0.50-0.67$ & $0.58 \pm 0.01,0.51-0.64$ & $0.62 \pm 0.02,0.53-0.77$ & $\begin{array}{l}F(2,33)=2.54 \\
p=.094\end{array}$ \\
\hline Familiarity & $0.81 \pm 0.02,0.66-0.91$ & $0.75 \pm 0.03,0.52-0.88$ & $0.79 \pm 0.02,0.69-0.89$ & $\begin{array}{l}F(2,33)=1.73, \\
p=.193\end{array}$ \\
\hline $\begin{array}{l}\text { Frequency } \\
\text { (days/week) }\end{array}$ & $2.47 \pm 0.41,0.67-6.15$ & $2.83 \pm 0.41,0.84-6.65$ & $2.33 \pm 0.45,0.74-6.09$ & $\begin{array}{l}F(2,33)=.38 \\
p=.689\end{array}$ \\
\hline Healthiness & $0.38 \pm 0.06,0.11-0.71$ & $0.49 \pm 0.04,0.29-0.76$ & $0.37 \pm 0.05,0.18-0.71$ & $\begin{array}{l}F(2,33)=1.75 \\
p=.189\end{array}$ \\
\hline $\begin{array}{l}\text { Expected } \\
\text { satiation }\end{array}$ & $0.46 \pm 0.05,0.22-0.76$ & $0.38 \pm 0.03,0.26-0.53$ & $0.36 \pm 0.03,0.21-0.56$ & $\begin{array}{l}F(2,33)=2.28 \\
p=.118\end{array}$ \\
\hline $\begin{array}{l}\text { Estimated energy } \\
\text { content(kcal) }\end{array}$ & $137.62 \pm 7.28,103.04-183.96$ & $127.54 \pm 4.66,113.07-160.31$ & $128.52 \pm 4.96,105.08-160.37$ & $\begin{array}{l}F(2,33)=0.93 \\
p=.403\end{array}$ \\
\hline $\begin{array}{l}\text { Estimated energy } \\
\text { density }\end{array}$ & $0.50 \pm 0.02,0.41-0.64$ & $0.61 \pm 0.01,0.54-0.70$ & $0.54 \pm 0.02,0.43-0.67$ & $\begin{array}{l}\mathrm{F}(2,33)=7.54, \\
p<.01 *\end{array}$ \\
\hline
\end{tabular}

400 
401 Table 6. Pearson $\mathrm{r}$ correlations and $\mathrm{p}$-values of all subjective ratings with participant

402 characteristics

403

\begin{tabular}{|c|c|c|c|c|c|c|c|c|c|c|c|c|c|c|}
\hline \multirow[t]{2}{*}{$\begin{array}{c}\text { Participant } \\
\text { characteristics }\end{array}$} & \multicolumn{2}{|c|}{ Liking } & \multicolumn{2}{|c|}{ Familiarity } & \multicolumn{2}{|c|}{$\begin{array}{l}\text { Expected } \\
\text { satiation }\end{array}$} & \multicolumn{2}{|c|}{ Frequency } & \multicolumn{2}{|c|}{ Healthiness } & \multicolumn{2}{|c|}{$\begin{array}{c}\text { Estimated } \\
\text { energy } \\
\text { content }\end{array}$} & \multicolumn{2}{|c|}{$\begin{array}{c}\text { Estimated } \\
\text { energy density }\end{array}$} \\
\hline & $r$ & $p$ & $r$ & $p$ & $r$ & $p$ & $r$ & $p$ & $r$ & $p$ & $r$ & $p$ & $r$ & $p$ \\
\hline BMI & .228 & .078 & -.159 & .221 & .018 & .892 & -.108 & .406 & .015 & .908 & .013 & .918 & .006 & .965 \\
\hline Body fat $\%$ & .01 & .942 & .106 & .418 & .04 & .759 & -.272 & .034 & -.155 & .232 & -.018 & .888 & -.211 & .102 \\
\hline Age & .024 & .857 & .385 & .026 & -.162 & .212 & -.228 & .077 & -.032 & .807 & -.079 & .543 & -.209 & .105 \\
\hline Hunger score & .129 & .324 & -.028 & .83 & .042 & .749 & .128 & .327 & .233 & .071 & .035 & .788 & .216 & .094 \\
\hline DFS & .29 & .023 & .017 & .895 & .095 & .465 & .473 & $<.001$ & .235 & .068 & -.065 & .62 & .311 & .015 \\
\hline Restraint & .277 & .031 & -.057 & .66 & .184 & .156 & .249 & .053 & -.139 & .285 & .047 & .717 & -.004 & .977 \\
\hline Emotional eating & 0.13 & .318 & .007 & .96 & -.028 & .828 & .193 & .136 & .04 & .758 & -.195 & .131 & .013 & .922 \\
\hline External eating & .378 & .003 & .192 & .138 & .047 & .72 & .071 & .588 & -.149 & .25 & -.121 & .351 & .175 & .177 \\
\hline
\end{tabular}


405 Table 7. Summary of food characteristics, visual properties, and perceptual ratings that were

406 similar $(p>0.1)$, trending to be different $(0.05 \geq p \leq 0.1)$, or different $(p<0.05)$ among the three

407 macronutrient categories (carb, fat, combo) according to between-category ANOVA

408 comparisons.

409

\begin{tabular}{|l|l|l|l|}
\hline & \multicolumn{1}{|c|}{ Similar } & \multicolumn{1}{|c|}{ Trending } & \multicolumn{1}{c|}{ Different } \\
\hline 1. Food characteristics & $\begin{array}{l}\text { Water } \\
\text { Energy density } \\
\text { Portion size } \\
\text { Price }\end{array}$ & $\begin{array}{l}\text { Sodium } \\
\text { Volume }\end{array}$ & $\begin{array}{l}\text { Carb* } \\
\text { Fat* } \\
\text { Protein }\end{array}$ \\
\hline 2. Visual properties & $\begin{array}{l}\text { Red } \\
\text { Green } \\
\text { Spatial frequency } \\
\text { Complexity }\end{array}$ & $\begin{array}{l}\text { Visual area } \\
\text { Blue }\end{array}$ & Brightness \\
\hline 3. Perceptual ratings & $\begin{array}{l}\text { Familiarity } \\
\text { Frequency } \\
\text { Healthiness } \\
\text { Expected satiation } \\
\text { Estimated energy content }\end{array}$ & Liking & Estimated energy \\
& density \\
\hline
\end{tabular}

411 Italicized variables highlighted in yellow are suggested for inclusion as covariates in analyses

412 that emerge from use with MacroPics. *Note that carb and fat content of the categories differed 413 by design. 


\section{References}

Asfaw, Abay. 2011. "Does Consumption of Processed Foods Explain Disparities in the Body Weight of Individuals? The Case of Guatemala.” Health Economics 20 (2): 184-95. https://doi.org/10.1002/hec.1579.

Beck, Aaron T., Norman Epstein, Gary Brown, and Robert A. Steer. 1988. “An Inventory for Measuring Clinical Anxiety: Psychometric Properties." Journal of Consulting and Clinical Psychology 56 (6): 893-97. https://doi.org/10.1037/0022-006X.56.6.893.

Brunstrom, Jeffrey M., Alex C. L. Drake, Ciarán G. Forde, and Peter J. Rogers. 2018.

Blechert, Jens, Anja Lender, Sarah Polk, Niko A. Busch, and Kathrin Ohla. 2019. "FoodPics_Extended-An Image Database for Experimental Research on Eating and Appetite: Additional Images, Normative Ratings and an Updated Review." Frontiers in Psychology 10 (March). https://doi.org/10.3389/fpsyg.2019.00307.

Blechert, Jens, Adrian Meule, Niko A. Busch, and Kathrin Ohla. 2014. "Food-Pics: An Image Database for Experimental Research on Eating and Appetite." Frontiers in Psychology 5 (June). https://doi.org/10.3389/fpsyg.2014.00617.

Boswell, Rebecca G., and Hedy Kober. 2016. "Food Cue Reactivity and Craving Predict Eating and Weight Gain: A Meta-Analytic Review." Obesity Reviews 17 (2): 159-77. https://doi.org/10.1111/obr.12354.

Brogden, Nina, and Eva Almiron-Roig. 2010. "Food Liking, Familiarity and Expected Satiation Selectively Influence Portion Size Estimation of Snacks and Caloric Beverages in Men.” Appetite 55 (3): 551-55. https://doi.org/10.1016/j.appet.2010.09.003. "Undervalued and Ignored: Are Humans Poorly Adapted to Energy-Dense Foods?" Appetite 120 (January): 589-95. https://doi.org/10.1016/j.appet.2017.10.015.

Brunstrom, Jeffrey M., and Peter J. Rogers. 2009. "How Many Calories Are on Our Plate? Expected Fullness, Not Liking, Determines Meal-Size Selection.” Obesity 17 (10): 1884 90. https://doi.org/10.1038/oby.2009.201.

Brunstrom, Jeffrey M., and Nicholas G. Shakeshaft. 2009. "Measuring Affective (Liking) and 

Appetite 52 (1): 108-14. https://doi.org/10.1016/j.appet.2008.09.002.

Buckley, Charlotte M., Sophie Austin, Bernard M. Corfe, Mark A. Green, Alexandra M.

Johnstone, Emma J. Stevenson, Elizabeth A. Williams, and Jeffrey M. Brunstrom. 2019. Adults." The Journal of Nutrition 149 (11): 2056-64. https://doi.org/10.1093/jn/nxz124.

Burger, Kyle S., Marc A. Cornier, Jan Ingebrigtsen, and Susan L. Johnson. 2011. “Assessing Food Appeal and Desire to Eat: The Effects of Portion Size \& Energy Density.” International Journal of Behavioral Nutrition and Physical Activity 8 (1): 101. https://doi.org/10.1186/1479-5868-8-101.

Canella, Daniela Silva, Renata Bertazzi Levy, Ana Paula Bortoletto Martins, Rafael Moreira Claro, Jean-Claude Moubarac, Larissa Galastri Baraldi, Geoffrey Cannon, and Carlos Augusto Monteiro. 2014. "Ultra-Processed Food Products and Obesity in Brazilian Households (2008-2009).” PLOS ONE 9 (3): e92752. https://doi.org/10.1371/journal.pone.0092752.

Charbonnier, Lisette, Floor van Meer, Laura N. van der Laan, Max A. Viergever, and Paul A. M. Smeets. 2016. "Standardized Food Images: A Photographing Protocol and Image Database." Appetite 96 (January): 166-73. https://doi.org/10.1016/j.appet.2015.08.041.

Cordain, Loren, S. Boyd Eaton, Anthony Sebastian, Neil Mann, Staffan Lindeberg, Bruce A. Watkins, James H. O’Keefe, and Janette Brand-Miller. 2005. “Origins and Evolution of the Western Diet: Health Implications for the 21st Century." The American Journal of Clinical Nutrition 81 (2): 341-54. https://doi.org/10.1093/ajcn.81.2.341.

DiFeliceantonio, Alexandra G., Géraldine Coppin, Lionel Rigoux, Sharmili Edwin Thanarajah, Alain Dagher, Marc Tittgemeyer, and Dana M. Small. 2018. "Supra-Additive Effects of Combining Fat and Carbohydrate on Food Reward." Cell Metabolism 28 (1): 33-44.e3. https://doi.org/10.1016/j.cmet.2018.05.018.

Fiszman, Susana, and Amparo Tarrega. 2017. "Expectations of Food Satiation and Satiety Reviewed with Special Focus on Food Properties.” Food \& Function 8 (8): 2686-97. https://doi.org/10.1039/C7FO00307B.

Forde, Ciarán G., Eva Almiron-Roig, and Jeffrey M. Brunstrom. 2015. "Expected Satiety: Application to Weight Management and Understanding Energy Selection in Humans." 
Current Obesity Reports 4 (1): 131-40. https://doi.org/10.1007/s13679-015-0144-0.

Francis, H., and R. Stevenson. 2013. "Validity and Test-Retest Reliability of a Short Dietary Questionnaire to Assess Intake of Saturated Fat and Free Sugars: A Preliminary Study.” Journal of Human Nutrition and Dietetics 26 (3): 234-42. https://doi.org/10.1111/jhn.12008.

Frost, Randy O., Gail A. Goolkasian, Robin J. Ely, and Fletcher A. Blanchard. 1982. “Depression, Restraint and Eating Behavior.” Behaviour Research and Therapy 20 (2): 113-21. https://doi.org/10.1016/0005-7967(82)90111-5. Charlotte Croese, Gabriel Bell, Giuliana Durighel, et al. 2009. "Fasting Biases Brain Reward Systems towards High-Calorie Foods.” European Journal of Neuroscience 30 (8): 1625-35. https://doi.org/10.1111/j.1460-9568.2009.06949.x.

Guillocheau, Etienne, Olga Davidenko, Agnès Marsset-Baglieri, Nicolas Darcel, Claire Gaudichon, Daniel Tomé, and Gilles Fromentin. 2018. "Expected Satiation Alone Does Not Predict Actual Intake of Desserts.” Appetite 123 (April): 183-90. https://doi.org/10.1016/j.appet.2017.12.022.

Han, Wenfei, Luis A. Tellez, Matthew H. Perkins, Isaac O. Perez, Taoran Qu, Jozelia Ferreira, Tatiana L. Ferreira, et al. 2018. "A Neural Circuit for Gut-Induced Reward.” Cell 175 (3): 665-678.e23. https://doi.org/10.1016/j.cell.2018.08.049.

Herman, C. Peter, and Janet Polivy. 1975. “Anxiety, Restraint, and Eating Behavior.” Journal of Abnormal Psychology 84 (6): 666-72. https://doi.org/10.1037/0021-843X.84.6.666.

King, Jill L., S. Nicole Fearnbach, Sreekrishna Ramakrishnapillai, Preetham Shankpal, Paula J. Geiselman, Corby K. Martin, Kori B. Murray, et al. 2018. "Perceptual Characterization of the Macronutrient Picture System (MaPS) for Food Image FMRI.” Frontiers in Psychology 9. https://doi.org/10.3389/fpsyg.2018.00017.

Labbe, David, Andreas Rytz, Nicolas Godinot, A. Ferrage, and Nathalie C. Martin. 2017. "Is Portion Size Selection Associated with Expected Satiation, Perceived Healthfulness or Expected Tastiness? A Case Study on Pizza Using a Photograph-Based Computer Task.” Appetite 108 (January): 311-16. https://doi.org/10.1016/j.appet.2016.10.012. Hedonic Scale.” Chemical Senses 34 (9): 739-51. https://doi.org/10.1093/chemse/bjp054. 
Louzada, Maria Laura da Costa, Larissa Galastri Baraldi, Euridice Martinez Steele, Ana Paula Bortoletto Martins, Daniela Silva Canella, Jean-Claude Moubarac, Renata Bertazzi Levy, et al. 2015. "Consumption of Ultra-Processed Foods and Obesity in Brazilian Adolescents and Adults." Preventive Medicine 81 (December): 9-15. https://doi.org/10.1016/j.ypmed.2015.07.018.

Mooreville, Mira, Lauren B. Shomaker, Samantha A. Reina, Louise M. Hannallah, L. Adelyn Cohen, Amber B. Courville, Merel Kozlosky, et al. 2014. "Depressive Symptoms and Observed Eating in Youth." Appetite 75 (April): 141-49. https://doi.org/10.1016/j.appet.2013.12.024.

Peirce, Jonathan W. 2007. "PsychoPy_Psychophysics Software in Python.” Journal of Neuroscience Methods 162 (1): 8-13. https://doi.org/10.1016/j.jneumeth.2006.11.017.

Poti, Jennifer M., Bianca Braga, and Bo Qin. 2017. "Ultra-Processed Food Intake and Obesity: What Really Matters for Health—Processing or Nutrient Content?" Current Obesity Reports 6 (4): 420-31. https://doi.org/10.1007/s13679-017-0285-4.

Prada, Marília, David Rodrigues, Margarida V. Garrido, and Joana Lopes. 2017. "Food-Pics-PT: Portuguese Validation of Food Images in 10 Subjective Evaluative Dimensions." Food Quality and Preference 61 (October): 15-25. https://doi.org/10.1016/j.foodqual.2017.04.015.

Provencher, Véronique, and Raphaëlle Jacob. 2016. "Impact of Perceived Healthiness of Food on Food Choices and Intake." Current Obesity Reports 5 (1): 65-71. https://doi.org/10.1007/s13679-016-0192-0.

Ren, Xueying, Jozélia G. Ferreira, Ligang Zhou, Sara J. Shammah-Lagnado, Catherine W. Yeckel, and Ivan E. de Araujo. 2010. "Nutrient Selection in the Absence of Taste Receptor Signaling." Journal of Neuroscience 30 (23): 8012-23. https://doi.org/10.1523/JNEUROSCI.5749-09.2010.

Rosenbaum, Diane L, and Kamila S White. 2015. “The Relation of Anxiety, Depression, and Stress to Binge Eating Behavior.” Journal of Health Psychology 20 (6): 887-98. https://doi.org/10.1177/1359105315580212.

Schneider, Caroline A., Wayne S. Rasband, and Kevin W. Eliceiri. 2012. "NIH Image to ImageJ: 25 Years of Image Analysis.” Nature Methods 9 (7): 671-75. https://doi.org/10.1038/nmeth.2089. 
Segnini, Sandra, Franco Pedreschi, and Petr Dejmek. 2004. "Volume Measurement Method of Potato Chips." International Journal of Food Properties 7 (1): 37-44. https://doi.org/10.1081/JFP-120022494.

Small, Dana M., and Alexandra G. DiFeliceantonio. 2019. "Processed Foods and Food Reward." Science 363 (6425): 346-47. https://doi.org/10.1126/science.aav0556.

Steele, Eurídice Martínez, Larissa Galastri Baraldi, Maria Laura da Costa Louzada, Jean-Claude Moubarac, Dariush Mozaffarian, and Carlos Augusto Monteiro. 2016. "Ultra-Processed Foods and Added Sugars in the US Diet: Evidence from a Nationally Representative Cross-Sectional Study.” BMJ Open 6 (3). https://doi.org/10.1136/bmjopen-2015-009892.

Tellez, Luis A., Sara Medina, Wenfei Han, Jozelia G. Ferreira, Paula Licona-Limón, Xueying Ren, TuKiet T. Lam, Gary J. Schwartz, and Ivan E. de Araujo. 2013. "A Gut Lipid Messenger Links Excess Dietary Fat to Dopamine Deficiency.” Science 341 (6147): 800802. https://doi.org/10.1126/science.1239275.

Tellez, Luis A., Xueying Ren, Wenfei Han, Sara Medina, Jozélia G. Ferreira, Catherine W. Yeckel, and Ivan E. de Araujo. 2013. "Glucose Utilization Rates Regulate Intake Levels of Artificial Sweeteners: Glucose Utilization and Artificial Sweeteners." The Journal of Physiology 591 (22): 5727-44. https://doi.org/10.1113/jphysiol.2013.263103.

van Strien, Tatjana, Jan E. R. Frijters, Gerard P. A. Bergers, and Peter B. Defares. 1986. "The Dutch Eating Behavior Questionnaire (DEBQ) for Assessment of Restrained, Emotional, and External Eating Behavior." International Journal of Eating Disorders 5 (2): 295315. https://doi.org/10.1002/1098-108X(198602)5:2<295::AIDEAT2260050209>3.0.CO;2-T.

Wilkinson, Laura L., Elanor C. Hinton, Stephanie H. Fay, Danielle Ferriday, Peter J. Rogers, and Jeffrey M. Brunstrom. 2012. "Computer-Based Assessments of Expected Satiety Predict Behavioural Measures of Portion-Size Selection and Food Intake.” Appetite 59 (3): 93338. https://doi.org/10.1016/j.appet.2012.09.007.

Zhang, Lingli, Wenfei Han, Chenguanlu Lin, Fei Li, and Ivan E. de Araujo. 2018. "Sugar Metabolism Regulates Flavor Preferences and Portal Glucose Sensing." Frontiers in Integrative Neuroscience 12. https://doi.org/10.3389/fnint.2018.00057. 